\title{
EL INTRAEMPRENDIMIENTO Y SU IMPACTO EN EL DESARROLLO DEL SECTOR DE AUTOPARTES COLOMBIANO
}

The intrapreneurship and its impact on the development of the colombian auto parts sector

RICARDO PRADA OSPINA

JULIO CÉSAR ACOSTA-PRADO

\section{RESUMEN}

Como efecto de la aceleración del cambio tecnológico, así como por la creciente competencia mundial de los últimos años, los directivos y empresarios han encontrado necesario adoptar un estilo de comportamiento de tipo emprendedor en la formulación de sus estrategias. Este capítulo pretende analizar el intraemprendimiento y su impacto en el desarrollo del sector de autopartes colombiano. Se trata de un estudio cualitativo que se realiza en tres fases: análisis, diseño e implementación del proceso de caracterización del emprendimiento corporativo en una muestra del sector de autopartes. En ese sentido, se realizó una revisión de la literatura para definir las variables intraemprendimiento y emprendimiento corporativo, y cómo se han desarrollado en las empresas.

Palabras clave: autopartes, desarrollo empresarial, intraemprendimiento.

\section{ABSTRACT}

As a result of the acceleration of technological change and the increasing global competition in recent years, managers and entrepreneurs have found it necessary to adopt an entrepreneurial style of behavior in the formulation of their strategies. This chapter aims to analyze the intrapreneurship and its impact on the development of the Colombian auto parts sector. This is a qualitative study that is carried out in three phases: analysis, design and implementation of the characterization process of the corporate enterprise in a sample of the auto parts sector. In that sense, a review of the literature was made to define the variables intrapreneurship and corporate entrepreneurship and how they have developed in companies.

Key words: auto parts, business renewal, intrapreneurship. 


\section{INTRODUCCIÓN}

Como efecto de la aceleración del cambio tecnológico, así como por la creciente competencia mundial de los últimos años, los directivos y empresarios han encontrado necesario adoptar un estilo de comportamiento de tipo emprendedor en la formulación de sus estrategias. Covin \& Slevin (1991) argumentan que las tres posturas emprendedoras -asunción de riesgos, innovación y proactividad-, desarrolladas en unidades de negocio nuevas e independientes, se pueden aplicar también a procesos corporativos.

El presente capítulo pretende analizar el intraemprendimiento y su impacto en el desarrollo del sector autopartes colombiano. Se trata de un estudio cualitativo que se realiza en tres fases: análisis, diseño e implementación del proceso de caracterización del emprendimiento corporativo en una muestra del sector de autopartes. En ese sentido, se realizó una revisión de la literatura para definir las variables intraemprendimiento y emprendimiento corporativo, y cómo se han desarrollado en las empresas.

Los resultados muestran que, a pesar de ser considerados el intraemprendimiento y el emprendimiento corporativo dos escenarios estratégicos de renovación empresarial, las empresas autopartistas colombianas no promueven su desarrollo estratégico, ya que asumen, principalmente, modelos de negocio tradicionales, basados en una orientación económica impulsada por factores básicos de producción.

Este capítulo se estructura de la siguiente forma: posterior a la introducción, el segundo epígrafe aborda el marco teórico, en donde se describen los constructos emprendimiento corporativo e intraemprendimiento, y se relaciona con el sector automotor, específicamente con el subsector de autopartes. El tercer 
epígrafe presenta el problema de investigación. En el cuarto se descubren los resultados y principales hallazgos encontrados, y por último, se establecen las consideraciones finales.

\section{FUNDAMENTACIÓN TEÓRICA: EMPRENDIMIENTO E INTRAEMPRENDIMIENTO}

El concepto de emprendimiento surge inicialmente de la unión de los vocablos francés entreprendre, y alemán unternehmen, que significan emprender (Veeraraghavan, 2009). Este vocablo emprendedor se asume como de origen francés y se refiere a quien asume riesgos, es decir, aquel que persigue beneficios y trabaja en equipo o individualmente, innovando, creando oportunidades de negocio (Hisrich, 1990; Bruneau \& Machado, 2006).

Originalmente emprendimiento se relaciona con capacidad empresarial, aun cuando en realidad es un término mucho más amplio. Say (1803) afirma que el entrepreneur, es decir, la persona que inicia una pequeña empresa, hace el cambio de recursos desde una zona de bajo rendimiento a una de productividad alta y de rendimiento mayor.

De cualquier forma, son numerosos los términos para describir los esfuerzos emprendedores dentro de las organizaciones ya establecidas, lo que en cierta medida ha entorpecido el desarrollo de este campo de investigación (Sharma \& Chrisman, 1999). De acuerdo con Coduras, Guerrero y Peña (2011), al igual que sucede con la mayoría de conceptos derivados del emprendimiento, no hay hasta el momento presente una definición consensuada y única de emprendimiento corporativo. Así, diversos autores como Morris \& Kuratco (2002), Hornsby, Kuratko y Zahra (2002), Phan, Orlichenko, Boyd, Angstadt, Coccaro, Liberzon 
\& Arfanakis (2009) y Parker (2011) han señalado que no existe un consenso en la terminología, lo cual provoca que se utilicen como sinónimos las siguientes expresiones:

- Intraemprendimiento (Susbauer, 1973; Pinchot, 1985; Knight, 1987).

- Emprendimiento corporativo (Burgelman, 1983; Zahra, 1991; Covin \& Slevin, 1991; Sharma \& Chrisman, 1999; Ireland, Hitt \& Sirman 2003; Kuratko, 2007; Parker, 2011).

- Emprendimiento estratégico (Ireland \& Webb, 2007; Kuratko, 2007).

El intraemprendimiento y el emprendimiento corporativo son considerados como sinónimos por varios investigadores. No obstante, Trujillo \& Guzmán (2008) mencionando a Åmo \& Kolvereid (2005), argumentan una diferencia conceptual entre emprendimiento corporativo e intraemprendimiento cuando sostienen que, aun cuando ambos términos están relacionados y representan procesos incrementales de renovación en las organizaciones, a través de iniciativas de innovación llevadas a cabo por los empleados, dichos términos representan ligeramente diferentes fenómenos de renovación organizacional. Según estos autores, la diferencia fundamental radica en que el intraemprendimiento está focalizado en las iniciativas independientes, o por cuenta propia de los empleados, y el emprendimiento corporativo por iniciativas internas de la empresa o por cuenta ajena.

Adicionalmente, el emprendimiento corporativo está relacionado con los procesos utilizados por la empresa para fomentar actividades de innovación a través de la iniciativa de los empleados; de igual manera, el impacto que sobre el éxito de la empresa puede tener el comportamiento de los individuos (Åmo \& Kolvereid, 2005).

Por el contrario, Stopford \& Badem-Fuller (1990) argumentan que 
el emprendimiento corporativo solo tiene lugar en el interior de la organización cuando describe el proceso de renovación de una empresa existente, llevado a cabo solo por actores de la misma. Considerando asi, el emprendimiento corporativo e intraemprendimiento podrían ser utilizados como sinónimos (Seshadri \& Tripathy, 2006).

Los emprendedores innovan para sí mismos, mientras que los intraemprendedores o emprendedores corporativos innovan para las empresas en las que trabajan. No obstante, la problemática está en que el espíritu emprendedor corporativo es un término abstracto (Lumpkin \& Dess, 1996). Schumpeter (1911) sostiene que la introducción de innovaciones por parte de los emprendedores podía darse a través de la creación de una nueva empresa o dentro de los límites de una organización existente. Sin embargo, a lo largo del tiempo este constructo ha variado en su alcance y significado, confundiéndose en la actualidad dos fenómenos interrelacionados dentro del mismo término: intraemprendimiento y emprendimiento corporativo.

Las diferentes definiciones permiten desprender que los emprendedores corporativos son, habitualmente, personas muy motivadas dentro de las organizaciones, con alta orientación al logro, proactivos en extremo, y que actúan con comodidad para tomar iniciativas que permitan alcanzar la realización o desarrollo de nuevos productos dentro de sus empresas (Prada \& Ocampo, 2014).

Para Guth \& Ginsberg (1990) el intraemprendimiento involucra la innovación en la formación de nuevos negocios y las transformaciones mediante renovación estratégica. Al igual que sucede con el emprendimiento en general, el de tipo corporativo está viviendo un momento de expansión y es objeto del interés de 
\& diversos agentes relacionados con el desarrollo económico, así como del cuerpo de investigadores de la creación de empresas.

Por esto, el intraemprendedor puede impactar en la organización a través de diversos resultados relacionados con diversos ámbitos de la misma (Morris \& Kuratko, 2002). Según Coduras et al. (2011), el impacto más evidente de este tipo de actividad es el desarrollo de nuevas empresas, ya sean de carácter interno o externo, siendo también muy relevante el diseño de nuevos modelos de negocio, que suelen generar valor $\mathrm{y}$ beneficios adicionales, así como el acceso a otros mercados.

\subsection{La industria automotriz}

El continuo proceso de cambio en el entorno económico, donde la aceleración de la globalización y el desarrollo de la economía del conocimiento vienen actuando como motores, dinamiza a las empresas de forma sistemática. Por ello, en la actualidad la prioridad de las empresas debería ser evaluar la posición competitiva y participación de sus productos, a fin de aprovechar las oportunidades del mercado que les permita fortalecerse frente a los retos de los nuevos competidores (Escolano \& Belso, 2003).

La industria automotriz no ha sido ajena a esta situación, lo que se evidencia con el cambio constante de los gustos y tendencias de los clientes, al igual que el comportamiento del mercado automotor. Machorro et al, (2009), argumentan que al contar con mayores opciones de compra los clientes se han vuelto más selectivos y naturalmente buscan obtener el mejor producto, en las mejores condiciones, y con el mejor trato. Por esto, a nivel mundial el sector automotor es muy competitivo y ha evolucionado paralelamente a la tecnología del producto, del proceso y de los materiales con los que se fabrican los automóviles. En Colombia, la globalización también ha generado cambios funda- 
mentales, no sólo en la economía, sino además en el comportamiento de los mercados y gustos de los clientes, específicamente en el sector automotor.

El sector automotor a nivel mundial, en las últimas décadas, se ha caracterizado por presentar un constante proceso de reestructuración, convirtiéndose en una de las industrias más dinámicas de la era moderna, generadora de efectos importantes en las distintas economías en términos de productividad, desarrollo tecnológico y competitividad.

La importancia del sector automotor en el desarrollo de un país radica en su capacidad de generar innovación en la adquisición y el desarrollo de tecnología, la gran generación de empleo, debido a las diferentes cadenas productivas de diversos sectores que lo integran, y el desarrollo que representa para la sociedad donde se desarrolla esta actividad (Calderón, Engle, Mora, Styner, Gómez, Zhu, 2011).

Con la finalidad de ocupar los primeros lugares en producción y ventas en los mercados mundiales, las empresas integrantes del sector han estado siempre en busca de innovaciones que les permitan fortalecer y hacer más competitivos sus procesos productivos (Carvajal, 2010). Este sector, siendo como es, dinámico, integra diferentes subsectores y componentes relacionados con el transporte terrestre motorizado, tales como, ensambladoras, autopartes, comercializadoras, talleres de servicio, motocicletas, centros de diagnóstico, entre otros.

Cuando se plantea identificar el estado actual del sector en Colombia, se propone conocer su importancia para el país. Según estudios del Sector Automotor Colombiano, realizados por la Superintendencia de Industria y Comercio en el año 2012, 
$\approx$ desde el punto de vista económico, se encontró que existe una correlación entre la venta de vehículos y la variación porcentual del Producto Interno Bruto real del país.

Esta correlación puede sustentarse en el consumo de vehículos nacionales. Según los datos suministrados por el Departamento Administrativo Nacional de Estadística (DANE, 2014), el 47,7\% de las unidades de vehículos comercializados fueron de producción nacional, el 52,3\% restante fueron de origen extranjero, es decir, en el mercado interno de vehículos se presenta una participación casi equitativa entre la cantidad de automotores importados y los fabricados en el país. Sin embargo, medido en pesos colombianos, las ventas de vehículos importados tienden a aumentar, mientras que las ventas de vehículos nacionales parecen permanecer constantes. Este incremento se explica por la reducción de las barreras de entrada y la generación de competencia basada en precio de venta unitario de automotores.

\subsection{Descripción del mercado automotriz en Colombia}

La industria automotriz en Colombia se centra principalmente en cuatro grandes actividades: ensamble de vehículos, producción de autopartes, comercialización de autos y ensamble de motocicletas; entre otros.

En Colombia, la cadena productiva del sector automotor se entiende como un conjunto de eslabones vinculados entre sí por relaciones de tipo proveedor-cliente-proveedor (Departamento Nacional de Planeación - DNP, 2004). 


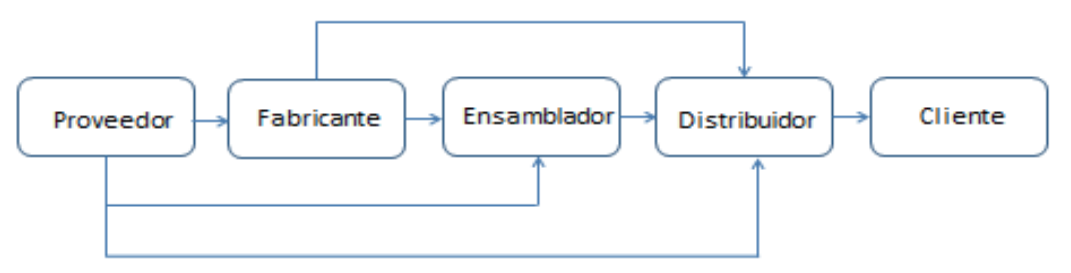

Figura 1. Cadena de valor del sector automotor

Fuente: Adaptado de Arango (2013)

De igual forma el Ministerio de Industria, Comercio y Turismo (2009), identificó que la cadena de valor del sector automotor en Colombia está integrada por proveedor, ensamblador, distribuidor, prestador de servicios post-venta o agente financiero (Zapata, Menendez, Prieto \& Romero 2013).

Por tanto, el proceso productivo del sector automotor comprende las actividades de ensamble de vehículos como la producción de partes, piezas y repuestos, las cuales también pueden ser utilizadas en el proceso de ensamble; es decir, comprende desde la fabricación de partes y piezas utilizadas en el ensamblaje hasta el ensamblaje de vehículos (vehículos ligeros, camiones, buses y motocicletas).

En Colombia se identifican siete empresas ensambladoras, dos de ellas de alta trayectoria, las cuales controlan el $54 \%$ del mercado nacional, distribuidas de la siguiente manera: GM Colmotores: $33,6 \%$ y Renault Sofasa: $15,0 \%$. Hasta agosto de 2014 existió además la Compañía Colombiana Automotriz (Mazda CCA), que controlaba el 5,4 \% del mercado nacional, pero dejó de operar definitivamente en cuanto a manufactura se refiere, dejando solo la operación de comercialización y venta.

Las restantes ensambladoras son: Hino Motors Manufacturing S.A., Carrocerías Non Plus Ultra, Compañía de Autoensamble Nissan, Navitrans S.A. y Daimler. Se debe señalar que las cuatro 
primeras empresas representan el $99 \%$ de la producción en términos de unidades (Calderón, Engle, Mora, Styner, Gómez \& Zhu, 2011; ANDI, 2013).

También hay que hacer notar que en la cadena automotriz en Colombia participan proveedores de insumos de otras industrias como la metalmecánica, la petroquímica (plásticos y cauchos) y la de textiles (BBVA, 2012; 2013) (Zapata et al, 2013).

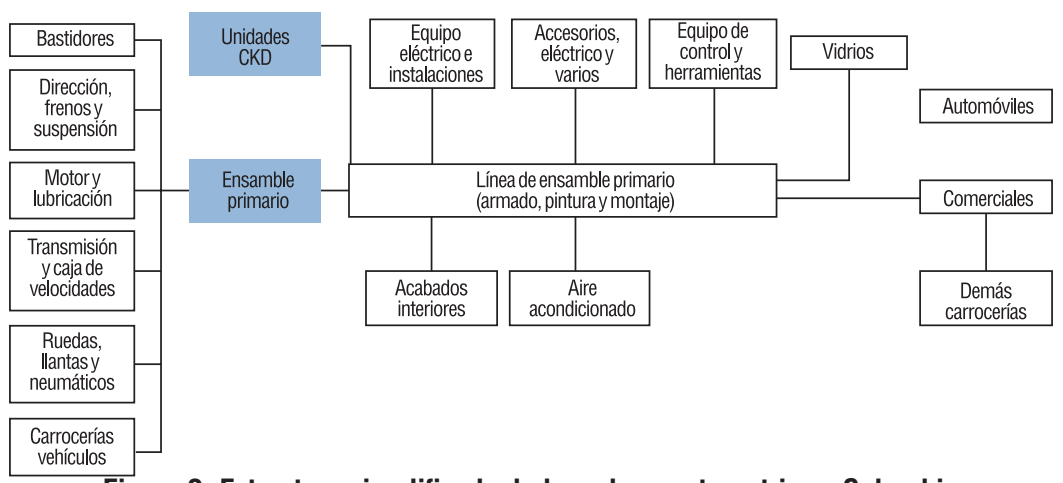

Figura 2. Estructura simplificada de la cadena automotriz en Colombia

Fuente: Dirección Nacional de Planeación - DNP (2007)

\subsection{El subsector de Autopartes}

En Bogotá se encuentra el parque automotor más grande de Colombia. Para 2013 alrededor de 1,8 millones de automóviles circulaban en la ciudad, esto representa el $39 \%$ del parque vehicular del país. Es un sector en expansión, ya que entre 2001 y 2010 el número de vehículos privados se incrementó en un $180 \%$ en la ciudad; además, Bogotá es sede de 6 de las 7 ensambladoras que tiene Colombia y el sector de autopartes, se concentra principalmente en tres ciudades: Bogotá, Cali y Medellín, empresas que, en su gran mayoría, están localizadas en Bogotá con cerca de 100 EMSA, que generan el $70 \%$ de la producción de autopartes del país (Invest in Bogotá, 2014). 
El sector de autopartes es el segundo eslabón de la cadena productiva que compone la industria automotriz colombiana. Sin embargo, aunque su participación se estima en menos del $20 \%$ de la producción total de este frente económico, detrás de él existen varios grupos que ejercen actividades de fabricación, comercialización y logística de estos productos, según lo concluido por el estudio "Caracterización integral de los procesos logísticos en el sector autopartes" (Zapata, Menendez, Prieto \& Romero, 2013).

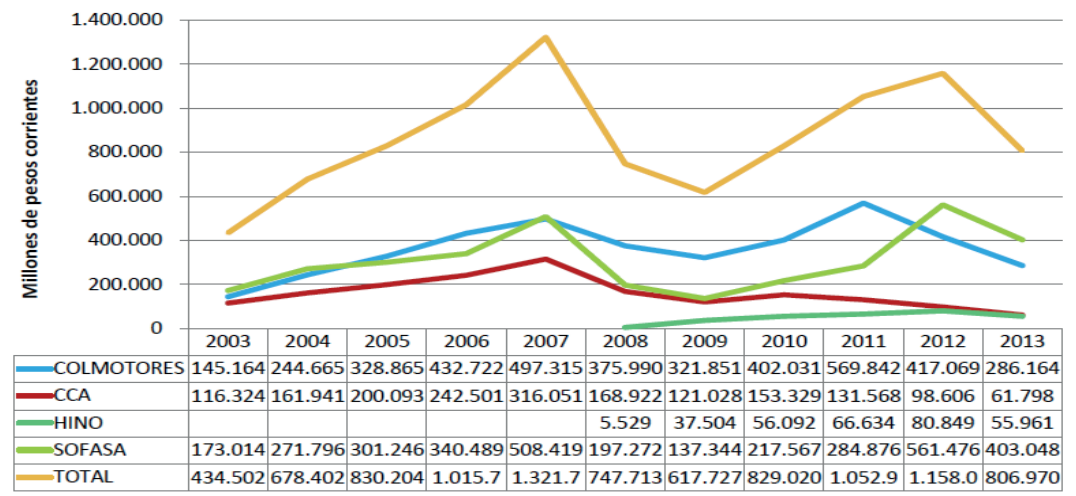

Figura 3. Compras de partes nacionales para ensamble

Fuente: ACOLFA (2014)

El sector de las piezas y partes para vehículos está compuesto por:

- $\quad$ Proveedores nacionales e internacionales que abastecen de piezas a fabricantes, ensambladores y distribuidores.

- Empresas fabricantes de piezas que suministran tanto a ensambladores como distribuidores.

- Empresas ensambladoras de vehículos ligeros, camiones, buses y motocicletas.

- Las sociedades distribuidoras, cuyo objeto social es la comercialización de autopartes.

También se incluyen las transportadoras de mercancías, que 
movilizan las piezas entre cada uno de los grupos anteriormente señalados. En cuanto a los subsectores por tipo de componente, según datos revelados por la Superintendencia de Industria y Comercio, para el año 2009 el 28 \% de la producción nacional de autopartes correspondió a equipos eléctricos e instalaciones; el $18 \%$ a sistemas de dirección, frenos y suspensión; y el $16 \%$ al de demás carrocerías, siendo estos los tres principales segmentos en la cadena productiva del sector de autopartes en Colombia.

Según Aguilar (2009) la baja sofisticación de las empresas conduce a improductividades de variada naturaleza. Es cuando se presenta poca incorporación de generación de valor por estrategias empresariales deficientes, así como por la presencia de desperdicios y la generación de costos innecesarios.

Con base en lo anterior, el objetivo principal de la presente investigación es caracterizar el intraemprendimiento y su impacto en el desarrollo del sector autopartes colombiano. Para ello, fueron consideradas las variables: innovación empresarial, capital humano, prácticas, procesos, implementación y resultados del intraemprendimiento, desde el nivel básico hasta el más alto. En consecuencia, se busca entender si las empresas medianas y grandes del sector de autopartes en la actualidad realizan o no actividades de intraemprendimiento, o son muy incipientes.

\section{METODOLOGÍA}

Autores como Katz (2010) han conceptuado en varios trabajos de investigación sobre la forma como el cambio estructural y las brechas tecnológicas destacan la importancia de los sectores difusores de conocimiento (SDC) en la dinámica económica como fuente de innovación del tejido productivo. Precisamente dentro de este SDC se encuentra el subsector metalmecánico, 
definiéndolo como aquel que genera bienes de capital y está relacionado con la creación de nuevas tecnologías, maquinaria y equipos para la industria y la transformación. Con base en esto, surge el interés de identificar si existen actividades de emprendimiento corporativo en este sector, aunque de manera informal.

Se trata de un estudio cualitativo que se realiza en tres fases: análisis, diseño e implementación del proceso de caracterización del emprendimiento corporativo en una muestra del sector de autopartes. La fase de análisis comprende el conocimiento teórico del emprendimiento corporativo y de sus variables intervinientes, así como del sector y su contexto. La fase de diseño implicó la construcción del instrumento a través del cual se reveló información sobre el emprendimiento corporativo, en una muestra de empresas del sector que accedieron a proporcionarnos sus datos. El instrumento fue diseñado para recopilar información productiva y económica de la empresa, innovación empresarial, capital humano, como variables contexto de la empresa para luego profundizar en el ciclo de desarrollo del emprendimiento corporativo: prácticas, procesos, implementación y resultados.

La muestra se obtuvo a partir del número de empresas legalmente constituidas en el sector de autopartes a 2015 en tres instituciones diferentes: Cámara de Comercio de Bogotá (CCB), Superintendencia de Sociedades (Supersociedades) y la Asociación Colombiana de Fabricantes de Autopartes (ACOLFA). En la CCB, bajo el código CIIU C2930, se registran 15 grandes empresas y 40 medianas, las cuales conforman el $70 \%$ del país (Araque, 2012).

La Supersociedades clasifica a las fabricantes de partes y repuestos para automotores mediante el código CIIU D3430, y las contabiliza en 115 empresas registradas; mientras que para ACOLFA son 38 las afiliadas. Al reunir los registros se encon- 
traron nombres de empresas coincidentes, las cuales se filtran para dar un total de 110 empresas legalmente registradas en las tres instituciones que conforman el marco muestral de la presente investigación. Se realizó un muestreo aleatorio, al enviar la encuesta a todas las empresas de marco muestral, de las cuales respondieron veinte con información completa (80 \%).

\section{RESULTADOS}

La Encuesta de Desarrollo e Innovación Tecnológica 2013-2014 que realiza el Departamento Administrativo Nacional de Estadística (DANE, 2014) ofrece información pertinente sobre las empresas que se dedican a la fabricación de vehículos automotores y sus partes. De allí se concluye que el $25 \%$ son innovadoras en sentido amplio y estricto, el 6,3\% potencialmente innovadoras y el $68,8 \%$ no innovadoras.

Del total de estas empresas, tan solo el $12,5 \%$ realizan innovaciones en el mercado nacional e internacional, y el resto innovaciones para la empresa. Es decir, a nivel nacional se encuentra que en el sector, aproximadamente el $40 \%$ realizan actividades innovadoras, por tanto, es probable que realicen procesos de emprendimiento corporativo.

En este capítulo se describe cómo de un total de 20 empresas del sector de autopartes que respondieron a la encuesta sobre intraemprendimiento, el $55 \%$ cuenta en nómina entre 10 y 49 empleados (empresas pequeñas, de acuerdo a la Ley 590 de 2000), el $30 \%$ entre 50 y 199 (empresas medianas), y sólo el $15 \%$, cuenta con más de 200 empleados (empresas grandes). No obstante, en la Tabla 1 se observa que el $55 \%$ de las empresas encuestadas declara ventas por más de 300 millones de pesos colombianos, el $60 \%$ tiene entre 5 y 10 años de antigüedad, y se extrae que el $70 \%$ forma parte de la industria manufacturera. Solamente el $10 \%$ de las empresas exporta. 
Tabla 1. Características de la muestra de empresas en el sector de autopartes

\begin{tabular}{|c|c|c|}
\hline \multicolumn{2}{|c|}{ COMPOSICIÓN DE LA MUESTRA } & SECTOR AUTOPARTES \\
\hline \multicolumn{2}{|c|}{ Tamaño de la muestra } & 20 \\
\hline \multicolumn{3}{|c|}{ Distribución de empresas por tamaño } \\
\hline & Muestra & \\
\hline & 10 y 49 & $55 \%$ \\
\hline & 50 y 199 & $30 \%$ \\
\hline & 200 o más & $15 \%$ \\
\hline \multicolumn{3}{|l|}{ Total ventas } \\
\hline & 51 y 100 millones & $30 \%$ \\
\hline & 101 y 300 millones & $15 \%$ \\
\hline & 301 millones o más & $55 \%$ \\
\hline \multicolumn{3}{|l|}{ Edad } \\
\hline & $<5$ años & $15 \%$ \\
\hline & 5 y 10 años & $60 \%$ \\
\hline & 10 o más años & $25 \%$ \\
\hline \multicolumn{3}{|l|}{ Industrial principal } \\
\hline & Manufactura & $70 \%$ \\
\hline & Servicios & $5 \%$ \\
\hline & Comercio & $25 \%$ \\
\hline
\end{tabular}

Fuente: Elaboración propia

\subsection{Características de las empresas}

Al preguntar sobre el uso de nuevas tecnologías, el $30 \%$ de las empresas encuestadas responde que no usa ninguna tecnología, el $55 \%$ que usa tecnología dura y el $15 \%$ tecnología blanda. El $45 \%$ indica que sí ha realizado procesos de innovación que han generado ingresos o disminución de costos, y de estos, mientras que el $70 \%$ son innovaciones de procesos, el $30 \%$ es innovación del servicio, como doblaje de láminas, en la producción en línea, en el almacenamiento múltiple de vehículos, y mercadeo y ventas. El $45 \%$ invierte menos del $25 \%$ de sus ingresos en nuevas tecnologías e innovación, el $35 \%$ entre 25 y $50 \%$, y el resto no reinvierte nada. 
\& Con relación a la distribución por género del talento humano que labora en las empresas analizadas, el $65 \%$ contrata en su mayoría más hombres (75\%) que mujeres (25\%). Al indagar por el nivel de formación educativa que contratan, se encuentra, en promedio, que el $46 \%$ de los empleados son trabajadores sin formación, pero con experiencia; el $27 \%$ cuenta con nivel técnico o tecnólogo; y el restante $27 \%$, lo conforma profesionales. Aproximadamente el $70 \%$ de los empleados en el sector de autopartes están contratados a término indefinido.

\subsection{Prácticas de intraemprendimiento}

Una vez que los gerentes de las empresas encuestadas fueron informados de lo que significa el emprendimiento corporativo, al preguntarles si creían que la empresa realizaba algún tipo de emprendimiento corporativo, el $45 \%$ responde que sí, el $45 \%$ que no y el restante $15 \%$ no sabe o no contesta.

Tabla 2. Empresas que realizan algún tipo de emprendimiento corporativo según el número de empleados en el sector de autopartes

\begin{tabular}{|c|c|c|c|}
\hline \multirow{2}{*}{ NÚMERO DE EMPLEADOS } & \multicolumn{3}{|c|}{$\begin{array}{c}\text { ¿SU EMPRESA REALIZA ALGÚN TIPO DE } \\
\text { ACTIVIDAD RELACIONADA CON EC? }\end{array}$} \\
\cline { 2 - 4 } & Sí & No & NS/NC \\
\hline Entre 10 y 49 empleados & 33,3 & 75,0 & 100 \\
\hline Entre 59 y 199 empleados & 44,4 & 12,5 & 0 \\
\hline Más de 200 empleados & 22,2 & 12,5 & 0 \\
\hline Total & 100 & 100 & 100 \\
\hline
\end{tabular}

Fuente: Elaboración propia

En la Tabla 2 se puede observar que del $100 \%$ de los encuestados que responden que su empresa realiza algún tipo de actividad relacionada con emprendimiento corporativo, el $33 \%$ son empresas pequeñas, el $45 \%$ medianas y el $22 \%$ grandes. 
Tabla 3. Razones por las que las empresas realizan o no actividades de emprendimiento corporativo

\begin{tabular}{|c|c|c|}
\hline \multirow[t]{2}{*}{ ¿POR QUÉ? } & \multicolumn{2}{|c|}{$\begin{array}{l}\text { ¿CREE USTED QUE SU EMPRESA } \\
\text { REALIZA ALGÚN TIPO DE ACTIVIDAD } \\
\text { RELACIONADA CON EC? }\end{array}$} \\
\hline & Sí & No \\
\hline Así como está funciona bien & 0 & 1 \\
\hline Así se comporta bien el negocio & 0 & 1 \\
\hline Creamos nuevas estrategias de ventas & 1 & 0 \\
\hline El tipo de negocio no permite incluir cambios & 0 & 1 \\
\hline Es un mercado específico ya establecido & 0 & 1 \\
\hline Investigación en tecnología de punta & 1 & 0 \\
\hline La actividad principal ya está establecida & 0 & 1 \\
\hline La empresa cree en el aporte de los trabajadores & 1 & 0 \\
\hline La labor es muy común & 0 & 1 \\
\hline $\begin{array}{l}\text { La línea de motos cambia constantemente en el mer- } \\
\qquad \text { cado }\end{array}$ & 1 & 0 \\
\hline Mejora en la venta y servicio oportuno & 1 & 0 \\
\hline Nuevas líneas de negocios con la dobladora & 1 & 0 \\
\hline Objeto social representación de una marca & 0 & 1 \\
\hline $\begin{array}{l}\text { Pensando en el crecimiento de la empresa y el incre- } \\
\text { mento de las utilidades }\end{array}$ & 1 & 0 \\
\hline Se cuenta con un departamento de innovación & 1 & 0 \\
\hline Se trata en mantener y atraer nuevos clientes & 1 & 0 \\
\hline Tiempo y negocio limitado & 0 & 1 \\
\hline
\end{tabular}

Fuente: Elaboración propia

Al preguntar a los gerentes de las empresas de autopartes, según la definición expuesta en el instrumento, si realizan algún tipo de actividad relacionada al emprendimiento corporativo, el $47 \%$ responde que sí la hace, a diferencia del $42 \%$ quienes responden que no, mientras el resto no contesta. Las que dicen que sí lo hacen aluden a su interés por mantener a los clientes, incrementar las ventas y mantenerse competitivos en el mercado; las que no lo hacen es porque perciben que el negocio es estático, se encuentran en su zona de confort, entre otras respuestas que se pueden observar en la Tabla 3. 
Al detallar el tipo de prácticas que las empresas están realizando en el marco del emprendimiento corporativo, como pregunta que confirme la respuesta si realizan prácticas de EC, se encuentra que a pesar que aproximadamente un $40 \%$ afirma que no realizan ninguna actividad, el $25 \%$ aproximadamente realiza trabajo colaborativo entre las diferentes áreas de la compañía; un $40 \%$ de manera frecuente realiza actividades orientadas al incremento de las ventas; el $15 \%$ actúa en grupos de trabajo para la generación de nuevas líneas de negocio (véase Tabla 4).

Tabla 4. Prácticas de Emprendimiento Corporativo

\begin{tabular}{|c|c|}
\hline $\begin{array}{l}\text { PRÁCTICAS PARA INVOLUCRAR A LOS EMPLEADOS EN EL FORTALECIMIENTO } \\
\text { DE LA EMPRESA }\end{array}$ & PORCENTAJE \\
\hline Generación de proyectos por equipos de trabajo & 10 \\
\hline Trabajo colaborativo entre diferentes áreas de la empresa & 25 \\
\hline Monitoreo y evaluación de oportunidades de negocio & 10 \\
\hline Ninguno & 55 \\
\hline Total & 100 \\
\hline PRÁCTICAS DE EMPRENDIMIENTO CORPORATIVO REALIZADAS DE MANERA FRECUENTE & PORCENTAJE \\
\hline Innovación de proceso, producto o servicio & 10 \\
\hline Creación o generación de nuevos productos & 10 \\
\hline Creación o generación de nuevas utilidades de negocio dentro de la empresa & 5 \\
\hline Búsqueda de nuevas oportunidades de negocio en el mercado & 35 \\
\hline Actividades orientadas al incremento de las ventas & 40 \\
\hline Total & 100 \\
\hline MECANISMOS PARA QUE LOS EMPLEADOS PROPONGAN NUEVAS LÍNEAS DE NEGOCIO & PORCENTAJE \\
\hline Incentivos a los empleados que proponen proyectos & 10 \\
\hline Grupos de trabajo para la generación de nuevas líneas de negocio & 15 \\
\hline Ninguno & 75 \\
\hline Total & 100 \\
\hline $\begin{array}{l}\text { RAZONES PARA NO REALIZAR NINGUNA PRÁCTICA DE APOYO } \\
\text { AL EMPLEADO EMPRENDEDOR }\end{array}$ & PORCENTAJE \\
\hline La empresa no lo requiere & 15 \\
\hline El clima organizacional no se adapta & 10 \\
\hline La estructura organizacional de la empresa no lo ha permitido & 25 \\
\hline Escasez de financiamiento & 15 \\
\hline Escasez de tiempo & 30 \\
\hline
\end{tabular}




\begin{tabular}{|c|c|}
\hline No le encuentra valor agregado a este tipo de prácticas & 5 \\
\hline Total & 100 \\
\hline RAZONES QUE HAN IMPEDIDO LA ADOPCIÓN DE PRÁCTICAS DE EMPRENDIMIENTO \\
CORPORATIVO & PORCENTAJE \\
\hline Riesgo corporativo & 25 \\
\hline Falta de recursos & 35 \\
\hline Bajo interés de las directivas & 35 \\
\hline Otro & 5 \\
\hline Total & 100 \\
\hline
\end{tabular}

Fuente: Elaboración propia

De acuerdo a la Tabla 4, se observa que los factores que más pesan para no realizar prácticas de emprendimiento corporativo son el bajo interés de las directivas y la falta de recursos específicos. Otros factores que se extraen de la Tabla son la escasez de tiempo para estas actividades y porque se cree que la estructura organizacional no lo permite.

\subsection{Procesos de emprendimiento corporativo}

A un nivel mayor de profundidad se cuenta con un grupo de preguntas que indagan sobre los procesos relacionados con el emprendimiento corporativo. En este campo el escenario es más precario que en el anterior. A la hora de profundizar en el trabajo en equipo y las propuestas de los empleados, el $55 \%$ afirma que no hace ningún proceso específico al respecto, y el $40 \%$ realiza reuniones para generación de ideas y propuestas de nuevas unidades de negocios, aunque $75 \%$ afirma que el gerente no se reúne con mandos medios y bajos a escuchar ideas de nuevos negocios, un $65 \%$ de las empresas indica que no se reflejan productos de estas actividades, y un $10 \%$ dice que se plasma en lluvia de ideas y en nuevos proyectos.

De las empresas que afirman realizar procesos de emprendimiento corporativo se indaga sobre el impacto en el mapa de procesos o la estructura organizacional, y el $90 \%$ afirma que no se ha observado un impacto a este nivel (véase Tabla 5). 
Tabla 5. Procesos de emprendimiento corporativo

\begin{tabular}{|c|c|}
\hline $\begin{array}{c}\text { ACTIVIDADES ESPECIFICAS QUE REALIZA LA EMPRESA CON RELACIÓN AL TRABAJO EN } \\
\text { EQUIPO Y A LAS PROPUESTAS DE SUS EMPLEADOS }\end{array}$ & PORCENTAJE \\
\hline Reuniones específicas para generación de ideas & 20 \\
\hline Generación de propuestas de nuevas unidades de negocio & 20 \\
\hline Otra & 5 \\
\hline Ninguna & 55 \\
\hline Total & 100 \\
\hline ¿EL GERENTE SE REÚNE CON MANDOS MEDIOS Y BAJOS PARA ESCUCHAR & PORCENTAJE \\
IDEAS DE NUEVOS NEEOCIOS? & 25 \\
\hline Sí & 75 \\
\hline No & 100 \\
\hline Total & PORCENTAJE \\
\hline LOS PRODUCTOS DE LAS ACTIVIDADES QUE GENERAN NUEVAS IDEAS & 10 \\
\hline Y PROYECTOS SE PLASMAN EN & 10 \\
\hline Lluvia de ideas & 10 \\
\hline Nuevos proyectos & 5 \\
\hline Nuevas unidades de negocio & 65 \\
\hline Otro & 100 \\
\hline Ninguna & \\
\hline Total & \\
\hline
\end{tabular}

Fuente: Elaboración propia

\subsection{Implementación del emprendimiento corporativo}

Otra fase de evaluación del emprendimiento corporativo es la implementación que se refiere a las actividades, indicadores relacionados a: la creación de nuevas unidades de negocio, la postura de las directivas en la fase de ejecución de nuevos proyectos, y el impacto que estos procesos de ejecución le han representado a la empresa. 
Tabla 6. Implementación del emprendimiento corporativo

\begin{tabular}{|c|c|}
\hline $\begin{array}{c}\text { ¿EN LOS ÚLTIMOS AÑOS CUÁNTAS LÍNEAS DE NEGOCIO, UNIDADES DE NEGOCIO O } \\
\text { NUEVOS PRODUCTOS SE HAN GENERADO? }\end{array}$ & PORCENTAJE \\
\hline Uno & 10 \\
\hline Menos de cinco & 25 \\
\hline Más de cinco & 5 \\
\hline Ninguno & 60 \\
\hline Total & 100 \\
\hline ¿CUÁL ES LA POSTURA DE LAS DIRECTIVAS ANTE LOS PROYECTOS? & PORCENTAJE \\
\hline Se quedan estancadas & 10 \\
\hline Las leen y evalúan su factibilidad & 30 \\
\hline Se implementan & 10 \\
\hline Otro & 50 \\
\hline Total & 100 \\
\hline $\begin{array}{l}\text { EN LA EMPRESA ¿SE HAN GENERADO NUEVOS PROCESOS, PRODUCTOS O } \\
\text { SERVICIOS EN LOS ÚLTIMOS AÑOS? }\end{array}$ & PORCENTAJE \\
\hline Sí & 30 \\
\hline No & 70 \\
\hline Total & 100 \\
\hline ¿CUÁL ES EL TIPO DE NOVEDAD? & PORCENTAJE \\
\hline Nuevos procesos & 20 \\
\hline Nuevos productos & 10 \\
\hline $\mathrm{NS} / \mathrm{NC}$ & 70 \\
\hline Total & 100 \\
\hline ¿CUÁNTAS UNIDADES DE NEGOCIO SE HAN IMPLEMENTADO EN SU EMPRESA? & PORCENTAJE \\
\hline Una & 20 \\
\hline Dos & 5 \\
\hline $\mathrm{NS} / \mathrm{NC}$ & 75 \\
\hline Total & 100 \\
\hline ¿QUÉ TIPO DE IMPACTO LE HA GENERADO EN SU EMPRESA? & PORCENTAJE \\
\hline Incremento en la productividad & 10 \\
\hline Incremento en los ingresos & 15 \\
\hline Reducción de costos & 10 \\
\hline Otro & 25 \\
\hline NS/NC & 40 \\
\hline Total & 100 \\
\hline
\end{tabular}

Fuente: Elaboración propia 
Es importante contar con una mirada integral del proceso de emprendimiento corporativo que da cuenta del tipo y la fase en el que se encuentra la empresa, es decir, si bien se encuentra que en el sector de autopartes se realizan prácticas y procesos de emprendimiento corporativo, en aproximadamente el $50 \%$ de ellas, reflejan cierta dinámica a la hora de verificar la implementación de esta apertura, nuevas ideas y su ejecución a través de la creación de nuevas unidades de negocio, se encuentra que aproximadamente el $40 \%$ ha generado alguna nueva unidad de negocio, línea de negocio o nuevos productos $10 \%$ uno, $25 \%$ menos de cinco y $5 \%$ más de cinco (véase Tabla 6 ).

A la hora de evaluar la postura de las directivas ante los proyectos o nuevas propuestas formuladas, el $50 \%$ de las empresas de autopartes indica que hay desinterés e indiferencia, $10 \%$ comenta que se quedan estancadas, el $30 \%$ indica que se leen y se evalúa su factibilidad y tan solo el $10 \%$ afirma que se implementan.

En el último año, el $30 \%$ de las empresas afirma que ha generado nuevo procesos, productos o servicios; de este porcentaje el $20 \%$ habla de una innovación de proceso y el $10 \%$ de producto. El impacto que ha representado la implementación del emprendimiento corporativo en aproximadamente el $40 \%$ de las empresas de autopartes se da en tres ámbitos: incremento en la productividad $(10 \%)$, incremento de los ingresos (15\%) y reducción de costos (10\%).

Todo esfuerzo de capital humano y recursos se debe evidenciar en resultados concretos que representen beneficios tangibles, como mayores ingresos, o intangibles, como el aprendizaje de la empresa. En este sentido, el apartado de medición de los resultados del emprendimiento corporativo tiene el objetivo de evidenciar los resultados de la ejecución de actividades del emprendimiento corporativo visto como impacto en el corto, mediano y largo plazo. 
Como se puede evidenciar en la Tabla 7, en la cual se incorporan las respuestas de las empresas del sector autopartes en cuanto a indicadores de emprendimiento corporativo a nivel organizacional, aproximadamente el $5 \%$ afirma contar con un equipo reconocido como emprendedores dentro la empresa, con una estrategia organizacional para la gestión de nuevas ideas, con bases de datos sobre ideas innovadoras, y con manuales de procedimiento para la gestión de ideas innovadoras.

El $95 \%$ de las empresas encuestadas no cuenta con indicadores de resultado del emprendimiento corporativo.

Tabla 7. Resultados del emprendimiento corporativo: organizacional

\begin{tabular}{|c|c|}
\hline $\begin{array}{c}\text { ¿EXISTE UN GRUPO IDENTIFICADO DE EMPRENDEDORES DENTRO DE SU } \\
\text { EMPRESA? }\end{array}$ & PORCENTAJE \\
\hline Sí & 5 \\
\hline No & 95 \\
\hline Total & 100 \\
\hline $\begin{array}{c}\text { ¿LA EMPRESA CUENTA CON UNA ESTRATEGIA ORGANIZACIONAL PARA LA } \\
\text { GESTIÓN DE NUEVAS IDEAS? }\end{array}$ & PORCENTAJE \\
\hline Si & 5 \\
\hline No & 95 \\
\hline Total & 100 \\
\hline ¿EXISTE UNA BASE DE DATOS PARA EL REGISTRO Y SEGUIMIENTO DE NUEVIAS \\
IDEAS O INNOVACIONES? & PORCENTAJE \\
\hline Sí & 10 \\
\hline No & 85 \\
\hline NS/NC & 5 \\
\hline Total & 100 \\
\hline ¿EXISTEN PROCESOS Y MANUALES DE PROCEDIMIENTOS DEFINIDOS PARA LA & PORCENTAJE \\
\hline CREACIÓN DE NUEVAS UNIDADES DE NEGOCIO? & 5 \\
\hline Sí & 90 \\
\hline No & 5 \\
\hline NS/NC & 100 \\
\hline TOtal & \\
\hline
\end{tabular}

Fuente: Elaboración propia 
En cuanto a la ejecución de recursos y financiamiento de los intraemprendimientos, se encuentra que el $65 \%$ no ofrece ningún incentivo, el $15 \%$ da incentivos verbales y $15 \%$ bonos. Al parecer, si la empresa cuenta con políticas e implementa nuevas unidades de negocio a partir de las ideas de sus emprendedores, estos se financian con recursos propios de la empresa en un $15 \%$, a través de convocatorias externas en un $5 \%$, endeudamiento $10 \%$ y el resto no contesta o no responde. A pesar del financiamiento que brinda el gobierno, el sector de autopartes lo considera solo en un $5 \%$ (véase Tabla 8).

Tabla 8. Resultados del intraemprendimiento: financiamiento

\begin{tabular}{|c|c|}
\hline $\begin{array}{c}\text { ¿CUÁLES SON LOS INCENTIVOS QUE OFRECE LA EMPRESA A SUS } \\
\text { INTRAEMPRENDEDORES? }\end{array}$ & PORCENTAJE \\
\hline Ninguno & 65 \\
\hline Incentivos verbales & 15 \\
\hline Bonos & 15 \\
\hline Otro & 5 \\
\hline Total & 100 \\
\hline ¿CUÁL ES EL TIPO DE FINANCIAMIENTO DE LAS NUEVAS UNIDADES DE & PORCENTAJE \\
\hline NEGOCIO GENERADAS POR SUS EMPLEADOS? & 15 \\
\hline Financiamiento propio de la empresa & 5 \\
\hline Convocatorias externas & 10 \\
\hline Endeudamiento & 60 \\
\hline Otro & 10 \\
\hline NS/NC & 100 \\
\hline Total &
\end{tabular}

¿CUÁL ES EL TIEMPO PROMEDIO TRASCURRIDO DESDE LA GENERACIÓN DE UNA IDEA HASTA SU IMPLEMENTACIÓN COMO BENEFICIO ECONÓMICO DE LA EMPRESA?

\begin{tabular}{|c|c|}
\hline Menos de 6 meses & 55 \\
\hline Entre 6 meses y 1 año & 20 \\
\hline Más de 1 año & 20 \\
\hline NS/NC & 5 \\
\hline Total & 100 \\
\hline $\begin{array}{c}\text { ¿QUÉ PORCENTAJE DE INCREMENTO TUVO LA EMPRESA EN SUS GANANCIAS } \\
\text { ATRIBUIDAS A LAS NUEVAS UNIDADES DE NEGOCIO CREADAS EN LOS } \\
\text { UULTIMOS 3 AÑNOS? }\end{array}$ & PORCENTAJE \\
\hline
\end{tabular}




\begin{tabular}{|c|c|}
\hline Ningún incremento & 65 \\
\hline Menos del $10 \%$ de incremento & 15 \\
\hline Entre el $11 \%$ y $20 \%$ & 15 \\
\hline Más del $20 \%$ & 5 \\
\hline Total & 100 \\
\hline $\begin{array}{c}\text { ¿QUÉ POSIBILIDADES DE SOSTENIBLIDAD A LARGO PLAZO TIENEN } \\
\text { ACTUALMENTE LAS NUEVAS UNIDADES DE NEGOCIO? }\end{array}$ & PORCENTAJE \\
\hline Baja & 75 \\
\hline Media & 15 \\
\hline Alta & 10 \\
\hline Total & 100 \\
\hline ¿LA EMPRESA SEGUIRÁ PROMOVIENDO NUEVAS UNIDADES DE NEGOCIO, & PORCENTAJE \\
\hline GENERADAS POR SUS EMPLEADOS, A LARGO PLAZO? & 35 \\
\hline Sí & 65 \\
\hline No & 100 \\
\hline Total & \\
\hline
\end{tabular}

Fuente: Elaboración propia

Tabla 9. Resultados del emprendimiento corporativo: eficiencia

¿CUÁL ES EL TIEMPO PROMEDIO TRASCURRIDO DESDE LA GENERACIÓN DE UNA IDEA HASTA SU IMPLEMENTACIÓN COMO BENEFICIO ECONÓMICO DE LA EMPRESA?

\begin{tabular}{|c|c|}
\hline Menos de 6 meses & 55 \\
\hline Entre 6 meses y 1 año & 20 \\
\hline Más de 1 año & 20 \\
\hline NS/NC & 5 \\
\hline Total & 100 \\
\hline
\end{tabular}

¿QUÉ PORCENTAJE DE INCREMENTO TUVO LA EMPRESA EN SUS GANANCIAS ATRIBUIDAS A LAS NUEVAS UNIDADES DE NEGOCIO CREADAS EN LOS ÚLTIMOS 3 AÑOS?

\begin{tabular}{|c|c|}
\hline Ningún incremento & 65 \\
\hline Menos del $10 \%$ de incremento & 15 \\
\hline Entre el $11 \%$ y $20 \%$ & 15 \\
\hline Más del $20 \%$ & 5 \\
\hline Total & 100 \\
\hline
\end{tabular}




\begin{tabular}{|c|c|}
\hline Baja & 75 \\
\hline Media & 15 \\
\hline Alta & 10 \\
\hline Total & 100 \\
\hline ¿LA EMPRESA SEGUIRÁ PROMOVIENDO NUEVAS UNIDADES DE NEGOCIO, \\
GENERADAS POR SUS EMPLEADOS, A LARGO PLAZO? & PORCENTAJE \\
\hline Sí & 35 \\
\hline No & 65 \\
\hline Total & 100 \\
\hline
\end{tabular}

Fuente: Elaboración propia

\section{CONCLUSIONES}

Los estudios de mercado muestran que la respuesta a las necesidades del cambio de deseos del cliente debe ser rápida. No obstante, con base en lo observado, se concluye que es bajo el porcentaje de empresas de autopartes que practican el emprendimiento corporativo. La respuesta a los tiempos que toma el desarrollo de un proyecto, desde la idea hasta su implementación, indica que el $55 \%$ de las empresas da respuesta en menos de 6 meses, el $20 \%$ en menos de un año y el $20 \%$ en más de un año (véase Tabla 9).

Las ganancias, atribuidas a nuevos proyectos, en los últimos tres años son aproximadamente menos del $10 \%$ de las utilidades para el $80 \%$ de las empresas de autopartes. Tan solo para el $5 \%$ representó más del $20 \%$.

En este escenario acelerado, al indagar sobre la sostenibilidad de las nuevas unidades de negocio, el $75 \%$ cree que son bajas, el $15 \%$ media y el $10 \%$ alta. Y, de acuerdo a todo lo anterior, tan solo el $35 \%$ de las empresas afirma que seguirán promoviendo el emprendimiento corporativo a largo plazo, mientras que el $65 \%$ afirma que no. 


\section{REFERENCIAS BIBLIOGRÁFICAS}

Acolfa (17 de septiembre de 2014). Desarrollo y prospectiva de la industria automotriz en Colombia. Obtenido de Desarrollo y Prospectiva de la Industria Automotriz en Colombia: http:// www.acolfa.org.co/index.php/acolfa/19-acolfa-2014/file.

Aguilar, C. (2009). Los subalternos como limitantes del poder. Hegemonía, legitimidad y dominación, en Contribuciones a las Ciencias Sociales. www.eumed.net/rev/cccss/04/craa. htm.

Åmo, B. \& Kolvereid, L. (2005). Estrategia organizativa, personalidad individual e innovación. Cultura Emprendedora (JEC), World Scientific Publishing Co. Pte. Ltd., 13(01), 7-19.

Antoncic, B. \& Hisrich, R. D. (2003). Clarifying the intrapreneurship concept. Journal of Small Business and Enterprise Development, 10(1), 7-24.

Arango, M. D. (2013). Caracterización integral de los procesos logísticos en el sector autopartes - automotores en Colombia - Propuesta Metodológica. Medellín.

Araque, N. (2012). Clasificación industrial uniforme de todas las actividades económicas. Bogotá: DANE. Obtenido de Informe de Coyuntura Económica Regional: http://www.dane.gov.co/ files/icer/2012/ICER_Bogota_Cundinamarca_2012.pdf.

Asociación Nacional de Empresarios de Colombia (2013). Automotriz y La ANDI. Disponible en: http://inflacion.com.co/ automotriz-y-la-andi-2013.html.

Banco Bilbao Vizcaya Argentaria (2012). Los ingresos de BBVA han crecido un 12,1\% en 2012 gracias a un modelo de negocio bien diversificado y equilibrado. Disponible en: https://accionistaseinversores.bbva.com/microsites/bbva2012/ es/index.html.

Banco Bilbao Vizcaya Argentaria (2013). BBVA Solidos Fundamentales. Disponible en:https://accionistaseinversores.bbva. com/microsites/bbvain2013/es/index.html.

Bruneau, J., Machado, H. (2006). Emprendedorismo en los países de América Latina, basado en los indicadores del Global Entrepreneurship Monitor (GEM). En: Panorama socioeconómico, 18-25.

Burgelman, R. (1983). A process Model of Internal CorporateVenturing in Diversified MajorFirm. Administrative ScienceQuarterly, 28, 223-244. 
Calderón, L., Engle, R., Mora, A., Styner, M., Gómez, G. \& Zhu, H. (2011). La exposición a la contaminación atmosférica urbana grave influye en los resultados cognitivos, el volumen cerebral y la inflamación sistémica en niños clínicamente sanos. Cerebro y Cognición, 77, 345-55.

Carvajal, J. (2010). Relaciones dirigentes-dirigidos: Un acercamiento desde los Derechos Humanos. Paipa, Colombia: II Congreso de Investigación en Administración.

Corduras, A., Guerrero, M. \& Peña, I. (2011). Emprendimiento Corporativo en España. GEM España-Fundacion Xavier De Salas para la dirección General de Política de la pyme.

Covin, J. G. \& Slevin, O. P. (1991). A conceptual model of entrepreneurship as firm behavior. Entrepreneurship Theory, and Practice, 16(1), 7-25.

Departamento Administrativo Nacional de Estadística (2014). Indicadores coyunturales. Disponible en: https://www. dane.gov.co/files/ses/ses_2014/Indicadores_Coyunturales_19_08_14.pdf

Departamento Nacional de Planeación (2007). Plan Nacional de Desarrollo 2006-2010. Estado Comunitario: desarrollo para todos. Bogotá: DNP.

Departamento Nacional de Planeación-FONADE (2004). Metodología para la Evaluación de la Formulación de los Planes de Desarrollo Municipales.

Departamento Nacional de Planeación (2004). Dirección de Estudios Económicos Programación Seminarios. Disponible en: https://colaboracion.dnp.gov.co/CDT/Estudios\%20 Econmicos/2004_Programaci\%C3\%B3n\%20a\%C3\%B1o.pdf

Drucker, P. F. (1985). Innovation and Entrepreneurship. USA: Harper \& Row.

Escolano, C. \& J. Belso (2003). Internacionalización y pymes: conclusiones para la actuación pública a partir de un análisis multivariante. Revista Asturiana de Economía, 27, 169-195.

Galán, J. L. \& Pizarro, I. (2005). El comportamiento emprendedor de las empresas: negocios internos y externos. Recuperado de http://catedradiversificacion.unizar.es/contenidos/publicaciones/ELCOMPORTAMIENTOEMPRENDEDOR.pdf

Gálvez E. J. (2011). Cultura intraemprendedora e innovación: un estudio empírico en las mipyme turísticas colombianas. Cuadernos de Administración, 27(46), 103-114. 
Gálvez, E. \& García, D. (2011). Cultura, innovación, intraemprendimiento y rendimiento en las mipyme de Colombia (Tesis doctoral). Universidad Politécnica de Cartagena.

Gálvez, E. J. \& García, D. (2011). Impacto de la Cultura Intraemprendedora en el rendimiento de las mipyme del sector turístico colombiano. Un estudio empírico. Universidad y Ciencia, 52, 27-36.

Garzón, M. (2008). Propuesta de modelo de emprendimiento corporativo o intraemprendimiento para la innovación. Revista Ciencias Administrativas. Universidad del Norte.

Guth, W. \& Ginsberg, A. (1990). Guest editor's introduction: Corporate entrepreneurship. Strategic Management Journal, 12, 5-15.

Hamel, G. (2009). El futuro de la administración. USA: Harvard Business School Press.

Hisrich, R. D. (1990). Entrepreneurship/Intrapreneurship. American Psychologist, 45(2), 209-229.

Hornsby, J. S., Kuratko, D. F. \& Montagno, R. V. (1999). Perception of internal factors for corporate entrepreneurship: A comparison of Canadian and U.S. managers. Entrepreneurship Theory and Practice, 24(2), 9-24.

Hornsby, J. S., Kuratko, D. F. \& Zahra, S. A. (2002). Middle managers' perception of the internal environment for corporate entrepreneurship: assessing a measurement scale. Journal of Business Venturing, 17(3), 253-274.

Invest in Bogotá (2014). Informe Anual: Promoción de Inversión. Disponible en: https://es.investinbogota.org/sites/default/ files/2017-11/informe-anual-2014_0.pdf

Ireland, D. R. \& Webb, J. W. (2007). Strategic entrepreneurship: Creating competitive advantage through streams of innovation. Business Horizons, 50(1).

Ireland, R., Hitt, M. \& Sirman, D. (2003). A model of strategic entrepreneurship: the construct and its dimensions. Journal of Management, 29(6), 963-989.

Jericó, P. (2013). ¡Necesitamos intraemprendedores! Expansión. com. Recuperado de: http://www.expansion.com/2013/01/18/ emprendedores-empleo/opinion/1358537150.html

Kantis, H. \& Drucaroff, S. (2011). Emprendimiento corporativo en América Latina, Conceptos, lecciones de la experiencia coreana y plataforma estratégica para su desarrollo en la región. Washington, D.C.: Fondo Multilateral de Inversiones (FOMIN). 
Kantis, H., Ishida, M. \& Komori, M. (2002). Empresarialidad en economías emergentes: Creación y desarrollo de nuevas empresas en América Latina y el Este de Asia. Inter-American Development Bank.

Katz, J. y Bernat, G. (2010) Micro to Macro interactions in the context of Argentine manufacturing activities: exit and entry of firms, productivity growth, structural change and innovative behavior in response to changes in the macroeconomic policy regime", paper presentado en Globelics 2010, $8^{\circ}$. Kuala Lumpur, Malaysia: International Conference, University of Malaya.

Knight, J. (1987). Foodservice Dictionary. New York: Van Nostrand Reinhold.

Kuratko, D. (2007). Corporate Entrepreneurship. Foundations and Trends in Entrepreneurship, 3(2), 1-51.

Kuratko, D. (2009). Entreprenuership Theory, Process, Practice. South-Wenster: Cenage Learning.

Kuz, D. (2010). Exploration of Intrapreneurship and Innovation in Advanced Technology Organizations in the Western United States. Recuperado de: http://gradworks.umi.com/3425617.pdf

Lessem, R. (1990). Gestión de la cultura corporativa. Madrid: Ediciones Díaz de Santos, S.A.

Ley (1803). Congreso de Colombia. Disponible en: http://www. secretariasenado.gov.co/senado/basedoc/ley_1803_2016.html.

Lombriser, R. (1994). Grandes intraempresarios: Desarrollo de nuevos negocios dentro de las organizaciones. Barcelona: Folio. S.A.

Lumpkin, G. T. \& Dess, G. G. (1996). Clarifying the entrepreneurial orientation construct and linking it to performance. Academy of Management Review, 21(1), 135-172.

Lumpkin, G. T. (2007). Intrapreneurship and Innovation, En J. R. Baum, M. Frese y R. A. Baron (Eds.), The Psychology of Entrepreneurship (237-263). New Jersey: Lawrence Erlbaum.

Lyon, D. W., Lumpkin, G. T. \& Dess, G. G. (2000). Enhancing entrepreneurial orientation research: operationalizing and measuring a key strategic decision making process. Journal of Management, 26(5), 1055-1085.

Machorro, A., Venegas, A., Resenos, E., et al. (2009). La calidad en el servicio como ventaja competitiva en una empresa automotriz. Academia Journals, Revista de Ingeniería Industrial, 3(1), 1-16. 
Manimala, M. J. (2006). Organizational Constraints on Innovation and Intrapreneurship: Insights from Public Sector. Vikalpa, 31, 49-60.

McDaniel, B. A. (2000). A Survey on entrepreneurship and innovation. The Social Science Journal, 37(2), 277-284.

Medina, C. \& Espinosa, M. (2000). La innovación en las organizaciones modernas. Revista Gestión y Estrategia, 5. Universidad Autónoma de México.

Ministerio de Comercio, Industria y Turismo (2009). DesarroIlando Sectores de Clase Mundial. Informe final Sector Textil, Confección, Diseño y Moda. Bogotá, D.C.

Morris, M. H. \& Kuratko, D. F. (2002). Corporate entrepreneurship. Orlando: Harcourt College Publishers.

Novoa, F. (2008). Intra-emprender... ¿Y qué es eso?. Revista de la Asociación de egresados del Inalde, 3, 17-23.

Parker, S. C. (2011). ¿Intrapreneurship or entrepreneurship? Journal of Business Venturing, 26(1), 19-34.

Phan, K. Orlichenko, A., Boyd, E., Angstadt, M., Coccaro, E., Liberzon, I., Arfanakis, K. (2009). Preliminary evidence of white matterab normality in the uncinate fasciculus in generalized social anxiety disorder. Biol Psychiatry, 66, 691-694.

Pinchot, G. (1985). Intrapreneuring (el empresario dentro de la empresa), cómo hacerse empresario sin salirse de la empresa. Bogotá: Norma.

Pinchot, G., Pellma, R. (1999). Intrapreneuring in action: a handbook for bussines innovation. San Francisco: Berrett-Koehler Publishers, inc.

Porter, M. (2007). Estrategia competitiva: técnicas para el análisis de los sectores industriales y de la competencia (1 ${ }^{\text {a }}$ ed.). México: Grupo Editorial Patria.

Prada, R. Ocampo, P. (2014). La productividad y la calidad y en su relación con el crecimiento en ventas. Caso de estudio: Compañía de Autoensamble en Colombia. Scientific Journal, 10(19), 1-10.

Prodem (2010). Programa de desarrollo emprendedor. Recuperado de http://www.prodem.ungs.edu.ar/blog/2010/12/ emprendimiento-corporativo/

Rothaermel, F. T., Agung, S. D. \& Jiang, L. (2007). University Entrepreneuship: a taxonomy of the literatura. Industrial and Corporate Change, 16(4), 691-791. 
Say, J. B. (1803). Tratado de Política Económica. México: Fondo de Cultura Económica.

Schumpeter, J. A. (1911). Theory of Economic Development. Oxford University Press. New York, 1961.

Seshadri, D. \& Tripathy, A. (2006). Innovation through intrapreneurship: The road less travelled. Vikalpa: The Journal for Decision Makers, 31(1), 17-29.

Sharma, P. \& Chrisman, J. J. (1999). Toward a reconciliation of the specific issues in the field of corporate entrepreneurship. Entrepreneurship Theory and Practice, 23, 3-11.

Stopford, J. \& Badem-Fuller, C. (1990). Creating corporate entrepreneurship. Strategic Management Journal, 15(7), 521-536.

Susbauer, J. (1973). Programa de emprendedurismo intra corporativo en la industria estadounidense. Universidad Estatal de Cleveland.

Trujillo, M. \& Guzmán, A. (2008). Intraemprendimiento: una revisión al constructo teórico, sus implicaciones y agenda de investigación futura. Cuadernos de Administración, 21(35), 37-63.

Veeraraghavan, V. (2009). Entrepreneurship and innovation. Asia-Pacific Business Review.

Vesga, R. (2007). Emprendimiento e innovación en Colombia: ¿Qué nos está haciendo falta? Recuperado el 20 de Noviembre de: http://web.unillanos.edu.co/docus/Emprendimiento\%20e\%20innovacion.pdf

Zahra, S. A. (1991). Predictors and financialoutcomes of corporate entrepreneurship: Anexploratory study. Journal of Business Venturing, 6(4), 259-285.

Zapata, A., Menendez, V. Prieto, M. \& Romero, C. (2013). A framework for recommendation in learning object repositories: An Example of application in Civil Engineering. Advances in Engineering Software, 56, 1-14. Available http://www.sciencedirect.com/science/article/pii/S0965997812001433. Lastaccessed April 09, 2016. 


\section{BIBLIOGRAFÍA CONSULTADA}

Antoncic, B. \& Hisrich, R. D. (2003). Clarifying the intrapreneurship concept. Journal of Small Business and Enterprise Development, 10(1), 7-24.

Departamento Nacional de Planeación - FONADE (2004). Metodología para la evaluación de la formulación de los Planes de Desarrollo Municipales.

Drucker, P. F. (1985). Innovation and Entrepreneurship. Harper \& Row.

Galán, J. L. \& Pizarro, I. (2005). El comportamiento emprendedor de las empresas: negocios internos y externos. Recuperado de http://catedradiversificacion.unizar.es/contenidos/publicaciones/ELCOMPORTAMIENTOEMPRENDEDOR.pdf

Gálvez E. J. (2011). Cultura intraemprendedora e innovación: un estudio empírico en las mipyme turísticas colombianas. Cuadernos de Administración, 27(46), 103-114.

Gálvez, E. \& García, D. (2011). Cultura, innovación, intraemprendimiento y rendimiento en las mipyme de Colombia (Tesis doctoral). Universidad Politécnica de Cartagena.

Gálvez, E. J. \& García, D. (2011). Impacto de la Cultura Intraemprendedora en el rendimiento de las mipyme del sector turístico colombiano. Un estudio empírico. Universidad y Ciencia, 52, 27-36.

Garzón, M. (2008). Propuesta de modelo de emprendimiento corporativo o intraemprendimiento para la innovación. Revista Ciencias Administrativas. Universidad del Norte.

Hamel, G. (2009). El futuro de la administración. Harvard Business School Press.

Jericó, P. (2013). Necesitamos intraemprendedores. Expansión. Com. Recuperado de: http://www.expansion.com/2013/01/18/ emprendedores-empleo/opinion/1358537150.html

Kantis, H. \& Drucaroff, S. (2011). Emprendimiento corporativo en América Latina, Conceptos, lecciones de la experiencia coreana y plataforma estratégica para su desarrollo en la región. Washington, D.C.: Fondo Multilateral de Inversiones (FOMIN).

Kantis, H., Ishida, M. \& Komori, M. (2002). Empresarialidad en economías emergentes: Creación y desarrollo de nuevas 
empresas en América Latina y el Este de Asia. Inter-American Development Bank.

Kuratko, D. (2009). Entreprenuership Theory, Process, Practice. South-Wenster: Cenage Learning.

Kuz, D. (2010). Exploration of Intrapreneurship and Innovation in Advanced Technology Organizations in the Western United States. Recuperado de: http://gradworks.umi.com/3425617. pdf

Lessem, R. (1990). Gestión de la cultura corporativa. Madrid: Ediciones Díaz de Santos, S.A.

Ley (1803). Congreso de Colombia. Disponible en: http://www. secretariasenado.gov.co/senado/basedoc/ley_1803_2016. html.

Lombriser, R. (1994). Grandes intraempresarios: Desarrollo de nuevos negocios dentro de las organizaciones. Barcelona: Folio. S.A.

Lumpkin, G. T. \& Dess, G. G. (1996). Clarifying the entrepreneurial orientation construct and linking it to performance. Academy of Management Review, 21(1), 135-172.

Lumpkin, G. T. (2007). Intrapreneurship and Innovation, En J. R. Baum, M. Frese y R. A. Baron (Eds.), The Psychology of Entrepreneurship (237-263). New Jersey: Lawrence Erlbaum.

Lyon, D. W., Lumpkin, G. T. \& Dess, G. G. (2000). Enhancing entrepreneurial orientation research: operationalizing and measuring a key strategic decision making process. Journal of Management, 26(5), 1055-1085.

Manimala, M. J. (2006). Organizational Constraints on Innovation and Intrapreneurship: Insights from Public Sector. Vikalpa, 31 , 49-60.

McDaniel, B. A. (2000). A Survey on entrepreneurship and innovation. The Social Science Journal, 37(2), 277-284.

Medina, C. \& Espinosa, M. (2000). La innovación en las organizaciones modernas. Revista Gestión y Estrategia, 5. Universidad Autónoma de México.

Pinchot, G., Pellma, R. (1999). Intrapreneuring in action: a handbook for bussines innovation. San Francisco: Berrett-Koehler Publishers, inc. 
Porter, M. (2007). Estrategia competitiva: técnicas para el análisis de los sectores industriales y de la competencia. ( $1^{\underline{a}}$ ed.). México: Grupo Editorial Patria.

Prodem (2010). Programa de desarrollo emprendedor. Recuperado de http://www.prodem.ungs.edu.ar/blog/2010/12/ emprendimiento-corporativo/

Rothaermel, F. T., Agung, S. D. \& Jiang, L. (2007). University Entrepreneuship: a taxonomy of the literatura. Industrial and Corporate Change, 16(4), 691-791. 